\title{
Complete Genome Sequence and Phylogenetic Analysis of Red Seabream Iridovirus Isolated From a Cage-Cultured Small Yellow Croaker (Larimichthys Polyactis) in China
}

\author{
Gengshen Wang \\ Zhejiang Ocean University \\ Wei Xu \\ Zhejiang Ocean University \\ Haoxue Cheng \\ Zhejiang Ocean University \\ Jianjun Xie \\ Zhejiang Ocean University
}

Aixu Bai

Huaian Customs District,China

Wenjun Xu

Zhejiang Ocean University

Qizhang Liang ( $\nabla 11817043 @ z j u . e d u . c n)$

Zhejiang University https://orcid.org/0000-0001-9080-1837

\section{Research Article}

Keywords: phylogenetic, iridovirus, croaker, RSIV

Posted Date: November 23rd, 2021

DOI: https://doi.org/10.21203/rs.3.rs-1060393/v1

License: (9) (i) This work is licensed under a Creative Commons Attribution 4.0 International License. Read Full License 


\section{Abstract}

Iridoviruses are emerging pathogens widely spread in diverse environments and hosts. Numerous species of the iridoviridae family are known to cause severe disease in freshwater and marine organisms. Here, we report the complete genome sequence and phylogenetic analysis of the iridovirus strain LPIV-ZS-2021 isolated from a small yellow croaker (Larimichthys polyactis) in China. The genome sequence comprises 110,560 bp with a $\mathrm{G}+\mathrm{C}$ content of $53.42 \%$, has 104 putative open reading frames (ORFs) and shares highest sequence homology with Red red seabream iridovirus (RSIV) isolated in Japan (98.61\%). Phylogenetic analysis revealed that it is clustered within RSIV clade1. This is the first fully sequenced RSIV genomes detected in the small yellow croaker. The host range expansion of Megalocytivirus genus iridovirus warrants further attention to determine its potential economic and ecological impact.

\section{Introduction}

The Iridoviridae gained broad public attention because they cause significant economic impact in aquaculture of ornamental and food fish[2, 7]. Based on the ATPase and major capsid protein (MCP) genes, members of the Iridoviridae family are classified into two subfamilies (Alphairidovirinae and Betairidovirinae) and six genera (Iridovirus, Chloriridovirus, Decapodiridovirus, Lymphocystivirus, Megalocytivirus, and Ranavirus) (https://talk.ictvonline.org/taxonomy/). The Iridoviridae family possesses broad species tropism and infect invertebrates[5] and poikilothermic vertebrates, such as fish [3, 13], amphibians[10, 12], and reptiles[4, 6, 10, 12]. Three genera of Iridoviridae, namely Ranavirus, Lymphocystivirus, and Megalocytivirus, have been shown to cause infectious disease with high morbidity and mortality in freshwater and marine fish[3, 11, 14].

In 2021, we observed high mortality of fish larvae with signs such as black body coloration, white gills and spleen and kidney enlargement in three cage-cultured small yellow croaker farms located in Zhoushan island, China. Tissue samples from the gills, spleen and kidney were collected from the fish exhibiting obvious symptoms that is typical in iridovirus infection. To better understand and characterize viral genomic features, whole genome sequencing was performed using next-generation sequencing technology. Genomic DNA library was prepared following standard procedure provided by lllumina and used for Illumina NovaSeq 6000 sequencing. After quality control was performed to remove adaptor reads, duplicate reads and host genomic sequences, trimmed reads were assembled using ABySS software (http://www.bcgsc.ca/platform/bioinfo/software/abyss). The assembled sequence was corrected using Gap Closer software (https://sourceforge.net/projects/soapdenovo2/files/GapCloser/). BLASTN searches of the assembled sequence revealed the high nucleotide sequence identity to the genomic sequence of members in the genus of Megalocytivirus. We named our strain as Larimichthys polyactis iridovirus ZS2021 (LPIV-ZS-2021) and deposited the full genome into Genbank under the accession no.

OL310752. The LPIV-ZS-2021 genome is presented in its full length of 110,560 bp and has a G+C content of $53.42 \%$. A total of 104 open reading frames (ORFs) were predicted coding proteins ranging from 45 to 1,169 amino acids on the sense (R) and antisense (L) DNA strands using Genome Annotation Transfer 
Utility (http://athena.bioc.uvic.ca/help/tool-help/help-books/genome-annotation-transfer-utility-gatudocumentation/) with the red sea bream iridovirus (RSIV) genome (GenBank accession no. AB104413.1) as a reference. Among the 104 putative genes, 30 genes were highly homologous to those of other iridoviruses and have defined functions (Figure.1).

Multiple sequence alignments results revealed that the genome sequence of LPIV-ZS-2021 has highest nucleotide acid sequence identity $(98.61 \%)$ with the red seabream iridovirus isolated in Japan, followed by the pompano iridovirus isolated from Florida pompano (Trachinotus carolinus) maricultured in the Caribbea Sea (98\% identity). To further examine the relationships between LPIV-ZS-2021 and other members in the Megalocytivirus genus, phylogenetic analyses were performed on the MCP gene sequences of iridoviruses available from Genbank database. Using the MEGA6 software with a neighborjoining method model (Jukes-Cantor model), 1000 bootstrap replicates and default parameters, a phylogenetic tree was constructed, which clearly shows the phylogenetic relationship between LPIV-ZS2021 and other megalocytiviruses. The results showed LPIV-ZS-2021 clustered within RSIV clade 1 (Figure.2).

The small yellow croaker, Larimichthys polyactis, is a benthopelagic marine fish species of the family Sciaenidae that is widely distributed in the Bohai, Yellow Sea and East China Sea of the Northwest Pacific Ocean[9]. The small yellow croaker is one of the most economically important marine fishery resource in China and East Asian countries because of its high meat quality and high capture production rate[8]. However, the wild resources of the small yellow croaker have severely decreased due to over-exploitation and deterioration of the environment in its native habitat[1]. Our study represents the first fully sequenced RSIV genomes detected in the small yellow croaker and is one of the earliest studies reporting iridoviruses infections in this species. Future studies are needed to determine the role of LPIV-ZS-2021 in disease and any negative impact it has on the mariculture and conservation of the small yellow croaker.

\section{Declarations}

Author contributions Conceptualization: XWJ and LQZ. Methodology: WGS, XJJ, XW and CHX. Software: WGS, BAX, Writing-review \& editing: LQZ. Funding acquisition: WGS, XJJ and XWJ.

Funding This study was supported by grants provided by the Zhejiang Major Agricultural Technology Cooperative Extension Project (2020XTTGSC04), The second batch of Zhoushan city special funds for the development of modern fishery in No. (2020)21 and National Key Research and Development Project (2020YFD0900803).

Conflict of interest The authors declare that they have no conflict of interest.

\section{References}

1. Chen W, Cheng Q (2013) Development of thirty-five novel polymorphic microsatellite markers inPseudosciaena polyactis (Perciformes:Sciaenidae) and cross-species amplification in closely 
related species, Pseudosciaena crocea.Biochemical Systematics \& Ecology47:111-115

2. Eaton HE, Ring BA, Brunetti CR (2010) The Genomic Diversity and Phylogenetic Relationship in the Family Iridoviridae.Viruses2:1458-1475

3. Hick P, Becker J, Whittington R (2016) Iridoviruses of Fish.Aquaculture Virology:127-152

4. Huang Y, Huang X, Liu H, Gong J, Ouyang Z, Cui H, Cao J, Zhao Y, Wang X-J, Jiang Y, Qin Q (2009) Complete sequence determination of a novel reptile iridovirus isolated from soft-shelled turtle and evolutionary analysis of Iridoviridae.BMC genomics10:224

5. Ince IA, Özcan O, Ilter-Akulke AZ, Scully ED, Özgen A (2018) Invertebrate Iridoviruses: A Glance over the Last Decade. Viruses 10

6. Johnson AJ, Pessier AP, Wellehan JF, Childress A, Norton TM, Stedman NL, Bloom DC, Belzer W, Titus VR, Wagner R (2008) Ranavirus infection of free-ranging and captive box turtles and tortoises in the United States.J Wildl Dis44:851-863

7. Kurita J, Nakajima K (2012)Megalocytiviruses. Viruses4:521-538

8. Li Y, Han Z, Song N, Gao TX (2013) New evidence to genetic analysis of small yellow croaker (Larimichthys polyactis) with continuous distribution in China.Biochemical Systematics \& Ecology 50:331-338

9. Li Z, Shan X, Jin X, Dai F (2011) Long-term variations in body length and age at maturity of the small yellow croaker (Larimichthys polyactis Bleeker, 1877) in the Bohai Sea and the Yellow Sea, China.Fisheries Research110:67-74

10. Papp T, Marschang RE (2019) Detection and Characterization of Invertebrate Iridoviruses Found in Reptiles and Prey Insects in Europe over the Past Two Decades. Viruses 11

11. Shi CY, Jia KT, Yang B, Huang J (2010) Complete genome sequence of a Megalocytivirus (family Iridoviridae) associated with turbot mortality in China. Virol J 7:159

12. Stöhr AC, López-Bueno A, Blahak S, Caeiro MF, Rosa GM, Matos APAd, An M, Alejo A, Marschang RE (2015) Phylogeny and Differentiation of Reptilian and Amphibian Ranaviruses Detected in Europe.PLoS ONE10:e0118633

13. Whittington RJ, Becker JA, Dennis MM (2010) Iridovirus infections in finfish - critical review with emphasis on ranaviruses. Journal of Fish Diseases33:95-122

14. Williams T, Bergoin M, van Oers MM (2017) Diversity of large DNA viruses of invertebrates.J Invertebr Pathol147:4-22

\section{Figures}




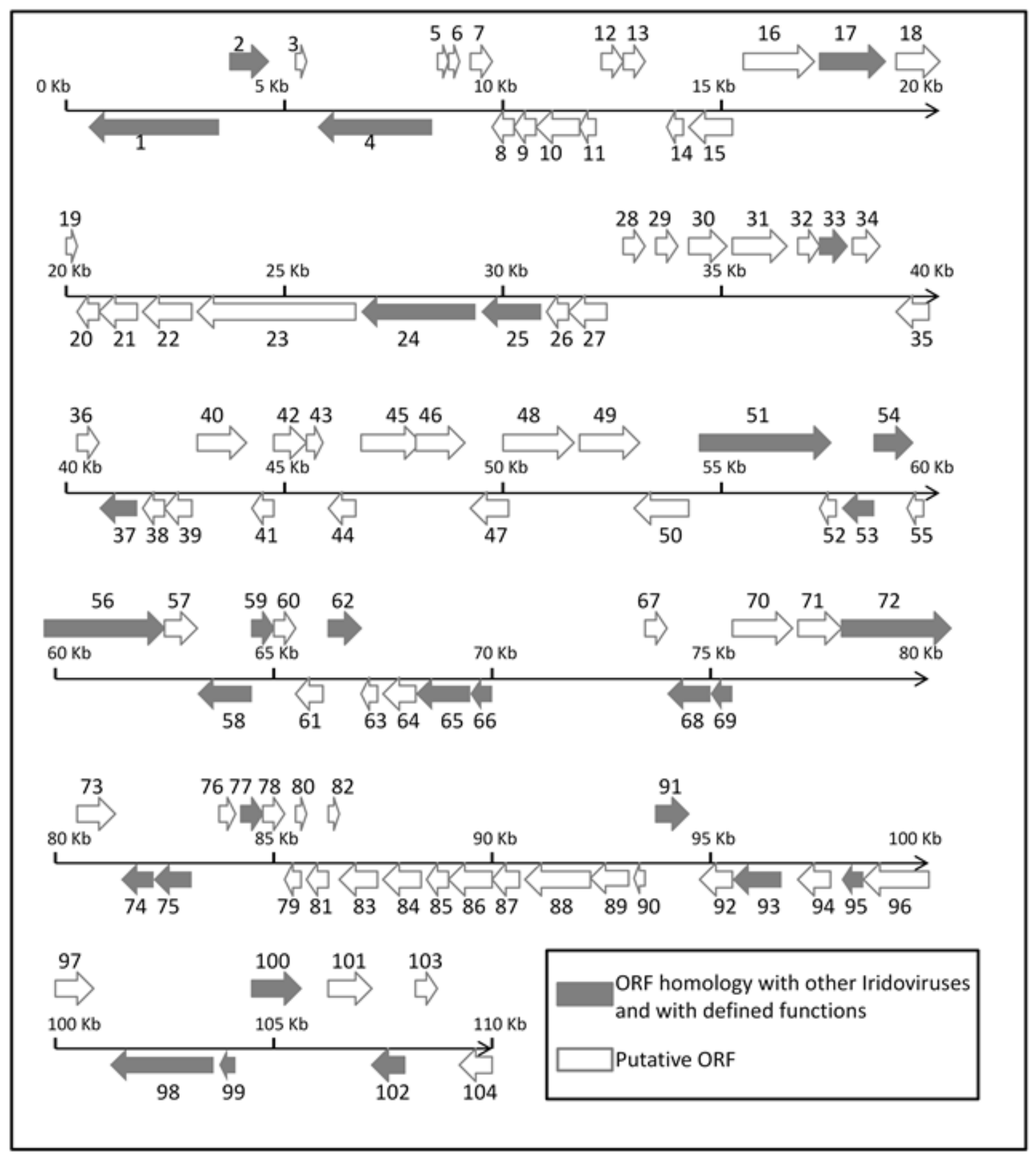

Figure 1

Schematic diagram of the LPIV-ZS-2021 genome. The LPIV-ZS-2021 genome is 110,560 bp in size and contains 104 potential open reading frames (ORFs). The scale presented here is in kilobase pairs. Arrows indicate the size, location and orientation of the ORFs. 


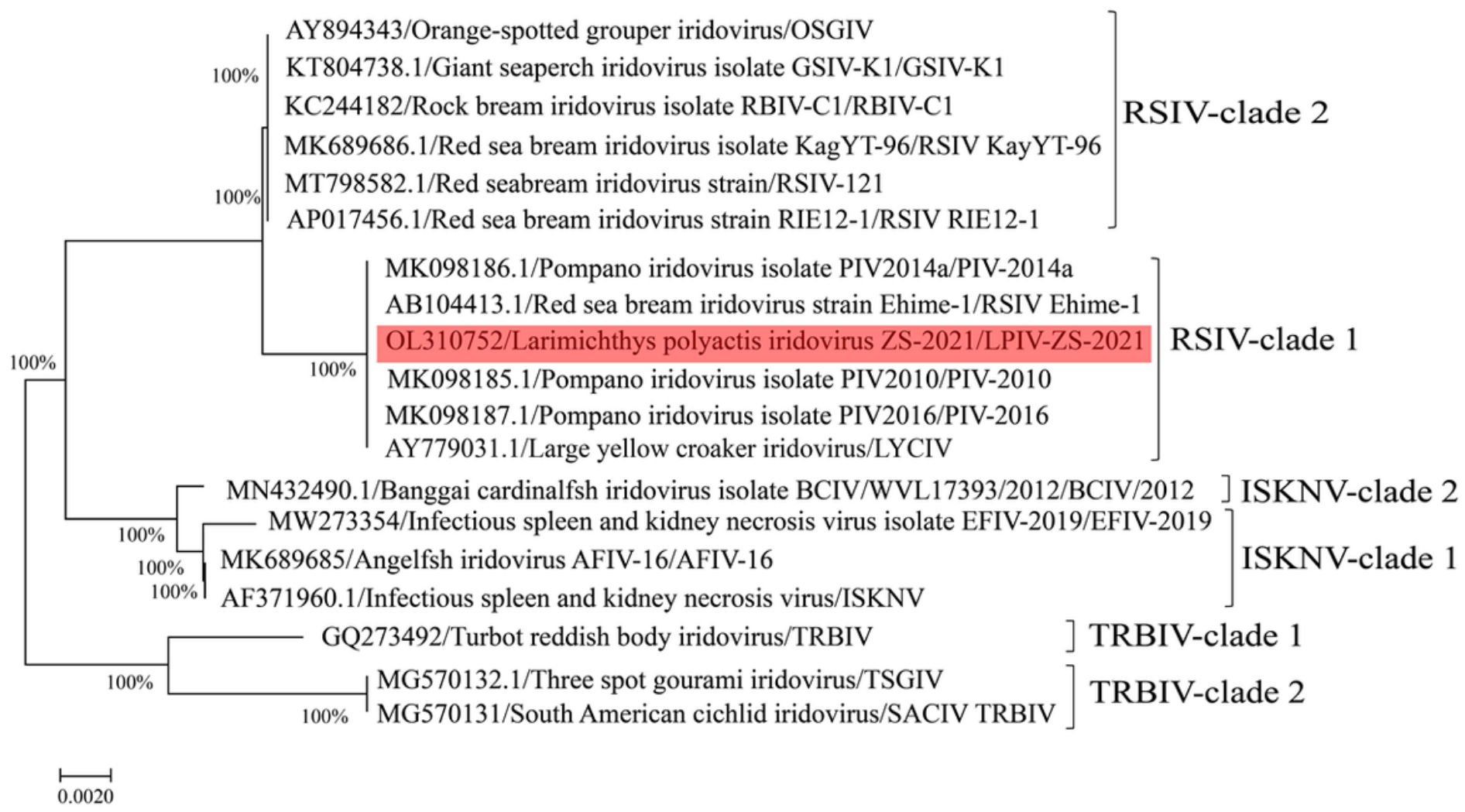

\section{Figure 2}

Phylogenetic analysis of MCP gene sequences of iridoviruses available through GenBank. The phylogenetic tree was constructed using the neighbor-joining method (MEGA-X). Bootstrap values are indicated at each node. The strain names and viral abbreviation are followed by GenBank accession numbers. LPIV-ZS-2021 is colored Red. 